\title{
Performance Analysis of an IoT Platform with Virtual Reality and Social Media Integration
}

\author{
Abhilash Krishnan*, Anderson Augusto Simiscuka ${ }^{\dagger}$ and Gabriel-Miro Muntean** \\ School of Electronic Engineering, Dublin City University, Dublin \\ Email: *abhilash.krishnan5@mail.dcu.ie, †anderson.simiscuka2@mail.dcu.ie, ${ }^{* *}$ gabriel.muntean@dcu.ie
}

\begin{abstract}
The Internet of Things (IoT) is a growing network of physical objects where the devices are connected to the Internet through unique addressing schemes and multiple protocols. The increase of IoT devices in the recent years presents significant challenges in terms of security, authentication and usability. The recently introduced Social Internet of Things (SIoT) tries to address these challenges with the virtualisation of IoT devices and the use of an infrastructure where people and IoT devices can communicate with each other, both in the real-world and virtual-world, through a common platform. In the proposed SIoT architecture, IoT devices can be operated by virtual reality (VR) headsets and Twitter, a social media platform. The aim of the platform is to allow users to seamlessly operate IoT devices, using their preferred interface: remotely with text messages (i.e. tweets) and VR headsets or operate the IoT devices directly. This paper also describes the implementation of a testbed and presents the performance analysis of the solution, demonstrating its feasibility and low latency.
\end{abstract}

Index Terms-Social Internet of Things (SIoT), Virtual Reality, Network Performance.

\section{INTRODUCTION}

The advances of the Internet of Things (IoT) in the recent years have created an ubiquitous computing vision, as everyday objects are embedded with computing features to create a socially interactive and smart environment. An IoT network needs to enable exchange of diverse data types including richmedia and sensor data and handle a large number of devices, offering services at high quality levels [1], [2], [3]. These goals are achieved by innovative solutions, which improve the performance and quality of IoT services and allow exchange of rich-media content [4], [5], [6].

The virtualisation of IoT devices allows the fulfilment of requirements of certain rich-media devices and platforms, such as virtual reality (VR) devices and social media, integrating them to the IoT network. In addition, certain IoT devices can be manipulated remotely in a virtual environment, as some devices can be complex to operate and do not provide a simple user experience. IoT devices can be also found in hazardous areas, such as landslide sensors and devices for water level monitoring, and VR can simulate these environments while communicating with the real devices [7].

In the context of IoT device virtualisation, a new paradigm known as the Social Internet of Things (SIoT) has emerged. SIoT aims to integrate social networking concepts into IoT networks, including a structure with guaranteed route navigation. It aims to ensure scalability and effective discovery

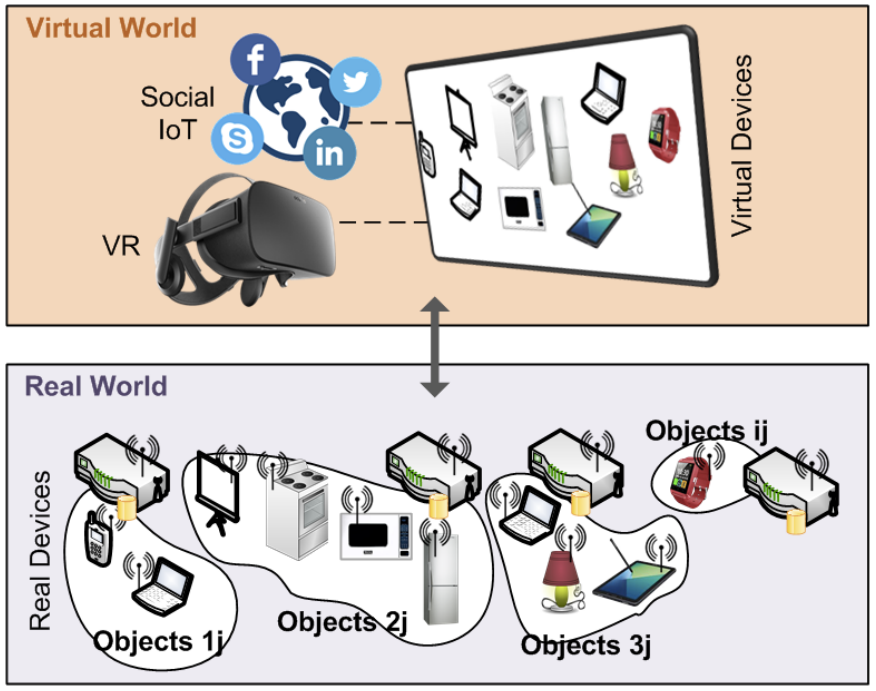

Fig. 1. Real-world and virtual-world devices

of connected objects and trust between connected objects and users that are 'friends' within the SIoT platform [8].

SIoT also introduces the ability to share the control of devices, which brings people together, and enables IoT services to be made accessible to users who do not possess certain devices. The possibility of manipulating virtual devices using intuitive controls and interfaces can make it easier for users to operate and interact with real IoT objects [9].

Social media platforms like Twitter and Facebook have been engaging millions of users across the globe for years now, with the number of users increasing every day. Twitter is a microblogging website with messages restricted to only 280 characters, which makes it a simple and practical interface for user and smart object interaction [10], [11].

Based on the concepts of SIoT, VR as an enabler for device manipulation and social media as a popular user interface, this paper presents a novel social IoT network which integrates the Twitter platform to allow users to control IoT devices remotely using text messages, as well as a VR environment that allows users to manipulate real devices in a 3D scenario [12], [13].

The solution, as illustrated in Fig. 1, maintains all platforms synchronised (i.e. VR platform, Twitter and real objects), therefore if a user tweets a command, real devices and the VR representation will receive the update. Similarly, operating 
a device from a VR headset will update the real device, and generate a tweet with the operation that was executed.

A real testbed was also built, with the Oculus Rift being used as the VR headset, Twitter as the social media interface, and a Raspberry Pi representing IoT devices. Testing includes the analysis of end-to-end delays and response times.

This paper is organised as follows. In section II, related works are presented and section III details the design and implementation of the testbed. Section IV presents testing and results. Section V finalises the paper indicating the conclusions and future work directions.

\section{RELATED WORKS}

The VR, social media and IoT-related research works described in this paper are divided into two categories: VR and IoT integration; and social media and IoT integration. These works inspired the solution presented in this paper which aims to integrate all three technologies for a seamless operation of IoT devices.

\section{A. VR and IoT Integration}

VR environments combine 3D spaces with a virtual representation of a user [14]. VR users are able to interact with different 3D objects, which are created using a 3D computer graphics software like Unity [15]. VR applications primary goal is to create a very immersive experience. Some VR headsets contain audio outputs, which might have a surround sound audio delivery system, like the Oculus Rift [16]. Oculus and HTC [17] are two of the major VR hardware manufacturers.

Based on the idea of creating innovative ways of controlling IoT devices, VR seems like a suitable approach due to the ever-increasing consumer adoption of VR technology.

Authors in [18] proposed the use of VR and Augmented Reality (AR) with IoT data streams from multiple locations in order to create a Mixed Reality (MR) environment, where users can collaborate, interact and navigate. The virtual environment displays digital data and the physical space is mapped for user navigation.

The work presented in [19] presents a cloud-based architecture where the interactions between real-world and virtualworld IoT devices are achieved through remote MQTT and REST low-latency communications. The researchers have analysed and compared the network performance parameters with a local-based approach, focusing on the synchronisation aspects of the implementation.

\section{B. Social Media and IoT Integration}

In [20], authors proposed a Web of Things architecture where the interaction between the humans and machines is achieved through the use of Twitter. The smart devices are connected to the Twitter API by smart gateways using the REST communication protocol. The researchers adopted the Web Service Business Process Execution Language (WSBPEL) for communications. The work, however, focuses more on surveying users than on hardware interaction.

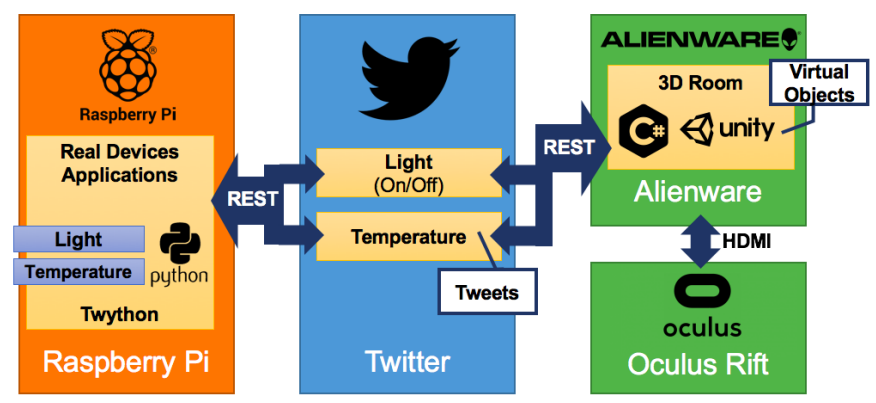

Fig. 2. High-level architecture

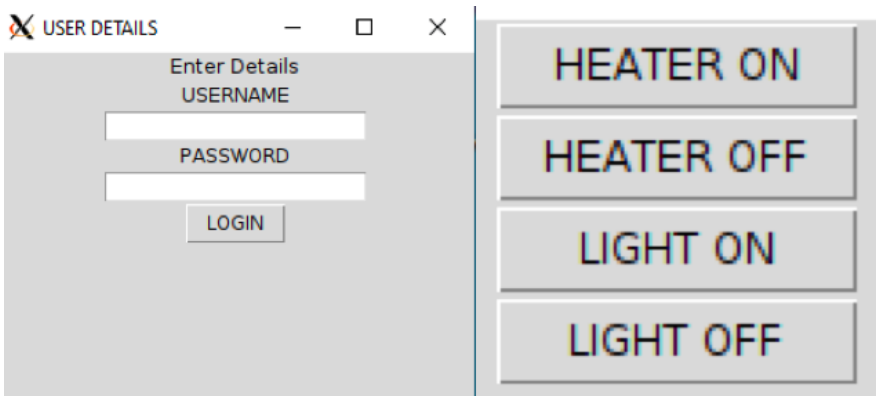

Fig. 3. Raspberry Pi interface

A Facebook-based SIoT network was proposed in [21]. The solution consists of a smart home environment to demonstrate the benefits of using social media features in IoT to monitor and control connected devices in a secure platform, such as Facebook. The work, however, did not present network analysis.

Waze [22] is also an example of a social IoT platform. Waze enables users to update traffic-related information in a collaborative application. It interconnects cars and users, and with the provided information, it helps drivers to find the best routes to get to their destination.

The solutions reviewed in this section provided insights to the implementation of our approach, such as the virtualisation of devices for manipulation through social media and VR, and the metrics that can be analysed for network performance. None of the reviewed works support both social media and VR integration to IoT in the same approach, therefore, there is a need of a research work that analyses the feasibility of such solution.

\section{DESIGN AND IMPLEMENTATION}

The high-level solution architecture is illustrated in Fig. 2. Design and implementation details are presented in this section. The implementation of the testbed allows for testing of the proposed architecture and solution performance analysis.

The overall solution consists of three components: the Raspberry Pi real-world implementation, the Unity-based virtualworld environment and the Twitter messaging solution. The three components are described in detail in this section. 


\section{A. Raspberry Pi Real-World Implementation}

The real-world IoT device in the testbed is the Raspberry Pi model 3B+, which has two functionalities: LEDs real-time control and CPU temperature status posted regularly to the Twitter timeline.

An application was developed in Python to connect the Raspberry Pi to the Twitter API's based on the REST protocol. Twitter requires the use of Consumer keys, Consumer Secret Keys, Access Token and Secret Access Token to authenticate the post actions from the Raspberry Pi. A GUI for login was created in the Raspberry Pi using python scripts to add extra security to the network, as seen in Fig. 3. The GUI contains buttons for controlling the Raspberry Pi locally: to turn on/off LEDs and temperature monitoring.

Users are required to enter a valid Twitter username and password to enable the GUI, which will post the live status of LEDs and CPU temperature to the Twitter timeline, ensuring that only authorised users can control the device. Users can be granted access to different functionalities of a device. For instance, a user might be allowed to visualise temperature statutes but not to interact with LEDs.

\section{B. Unity-Based Virtual-World Environment}

Unity is a cross-development game platform developed by Unity Technologies, and it supports the Oculus Rift and HTC Vive VR headsets, providing tools for the creation of immersive 3D environments. The Oculus Rift was the VR headset used for testing the virtual environment developed. The headset was connected to a Dell Alienware computer which has Unity installed, as well as the Oculus application.

A 3D room was developed in Unity with assets provided by the platform, as seen in the screen capture from Fig. 4. The main camera object, directional lights, point lights, cubes and planes were used in the creation of the 3D scene. The effect of lights in the scene was achieved through point lights controlled by the programming logic.

The requests from the Unity application to Twitter were made using POST URLs, which are available on the Twitter Developer help center. These requests were implemented in $\mathrm{C \#}$ scripts that are executed when users interact with the $3 \mathrm{D}$ objects, using the Oculus Touch controllers.

The requests contain messages with several fields: date, time, game object name, status of the game object and Twitter hashtag. For instance, when the virtual light bulb is switched on or off in the VR environment, a message with the aforementioned fields is displayed on the Twitter timeline. The tweet will result on the real LED being turned on or off in the Raspberry Pi.

Similarly to the Raspberry Pi application, users are required to login in the VR application, in order for them to have the right access level, as illustrated in Fig. 5.

The VR application is also updated when users tweet. The GET URL provided by Twitter is used to retrieve messages

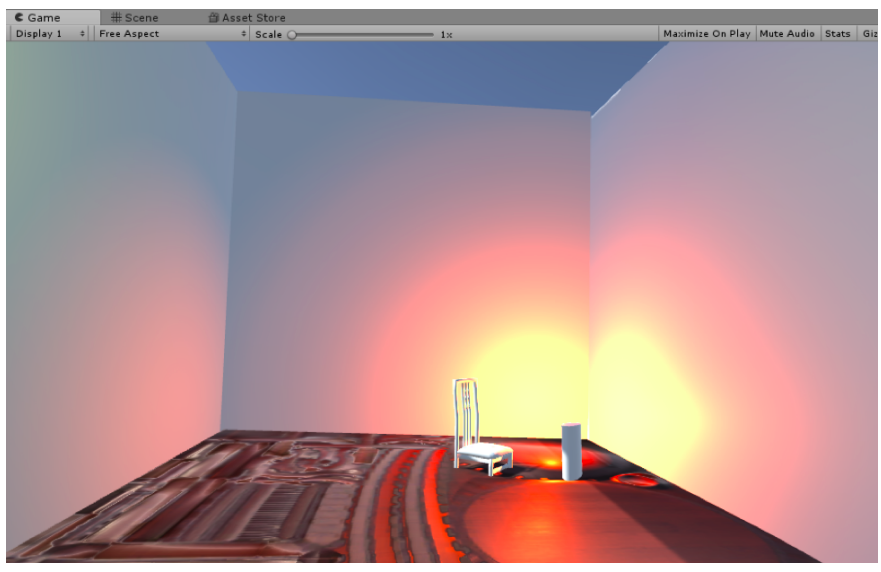

Fig. 4. Virtual environment - light on

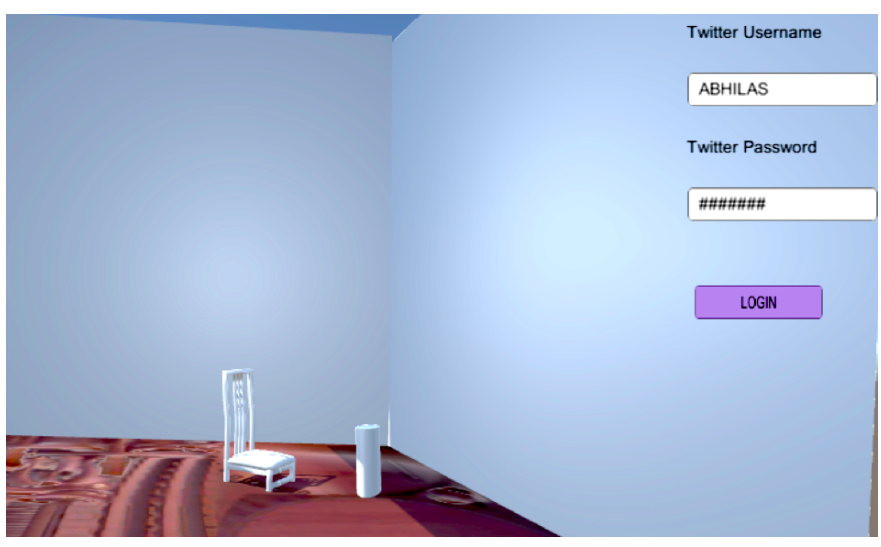

Fig. 5. Virtual environment - light off and login form

from the Twitter timeline. Game objects in the VR environment constantly verify the Twitter timeline and update their statuses following the actions required by users. For instance, the tweet "LIGHT ON \#[hashtag]" will result on the light turning on in the VR environment, as well as the LED on the Raspberry Pi.

\section{Twitter Messaging Solution}

The Raspberry Pi accesses the Twitter timeline with the Twython Streamer package [23], which is executed in the background and keeps polling the user's tweets generated by the user on Twitter or on the VR environment.

For instance, the tweet "LIGHT ON \#[hashtag]" generated by an action on the VR environment or typed by the user, is retrieved by the Twython streamer based on the hashtag. The Raspberry Pi Python application will, then, change the state of the LED on the Raspberry Pi using the GPIO commands.

The communications of the Raspberry Pi real-world implementation with the Unity-based virtual-world environment and with the Twitter messaging solution, is also illustrated in the sequence diagram presented in Fig. 6 . 


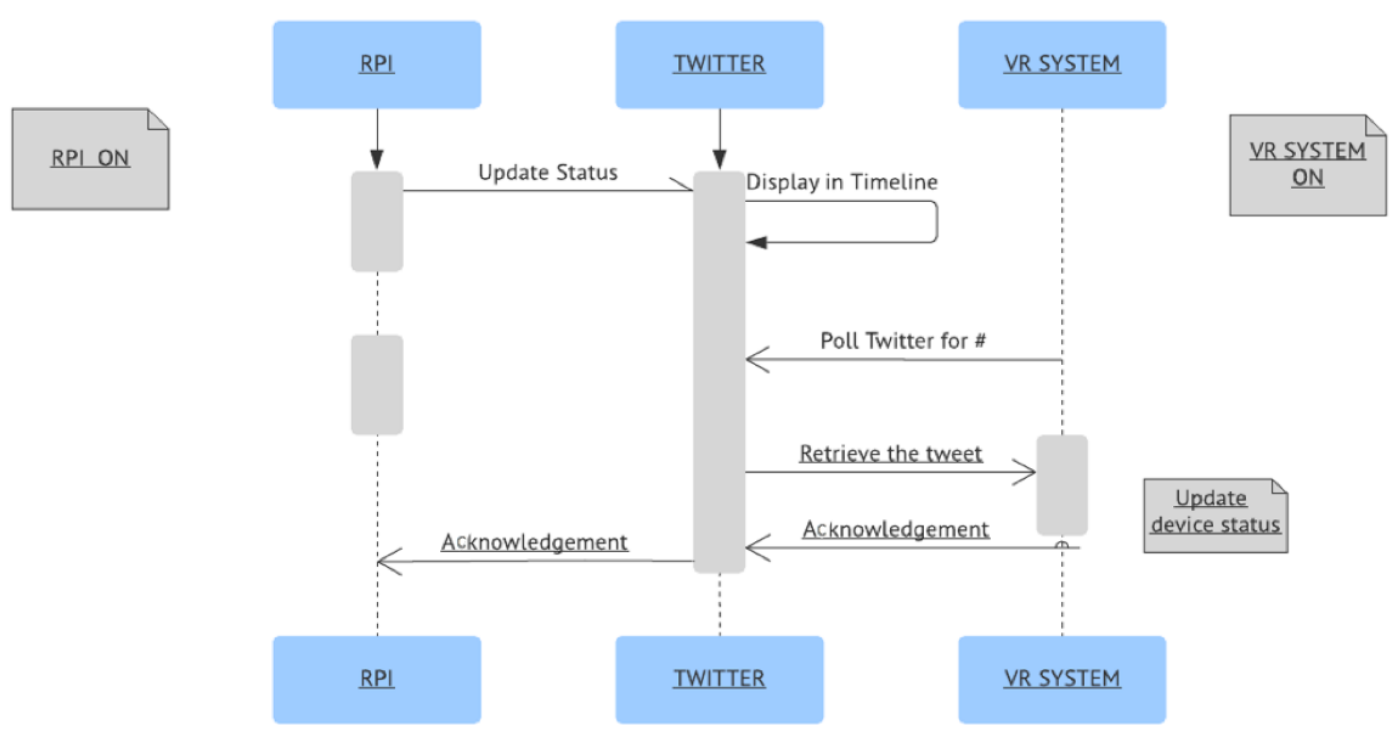

Fig. 6. Sequence diagram of the solution

\section{RESULTS AND DISCUSSION}

For performance analysis, the application was tested in terms of tweeting delay (i.e. the time an action executed in the Raspberry Pi or VR environment took to be tweeted), and in terms of end-to-end delay, which considered the time for an action to be tweeted and also updated in all systems (i.e. Raspberry Pi and VR environment). Wireshark was used for the measurements, as it is a useful tool for detecting the packets used in the communication.

The application was executed ten times, and in each time, the delay was measured. This allowed for an estimate of minimum, average and maximum delays, for both tweeting delay and end-to-end delay.

\section{A. Tweeting Delay}

The tweeting delay was measured in relation to the time taken by an action executed in the VR environment or Raspberry Pi to be tweeted with the Twitter REST API. The tweeting delay was also compared against the solution presented in [19], which used Adafruit IO as a cloud-based server for registering user actions from a VR-only platform integrated with IoT. In this paper, Twitter is the "cloud platform", as the tweets are main avenue for communication between the IoT device and the users (on Twitter and on the VR environment).

The plot in Fig. 8 shows that the Twitter REST API performed better than the Adafruit REST API in the ten test executions. The minimum, average and maximum delays observed in the Twitter REST API were 0.351s, 0.442s and 0.768s respectively, while for the Adafruit REST API these values were $0.439 \mathrm{~s}, 0.826 \mathrm{~s}$ and $1.353 \mathrm{~s}$, respectively. Therefore, the Twitter REST API has, in average, 53\% less delay than the Adafruit REST API.

The Adafruit MQTT API, however, has a smaller delay in comparison to the Twitter REST API, with an average delay

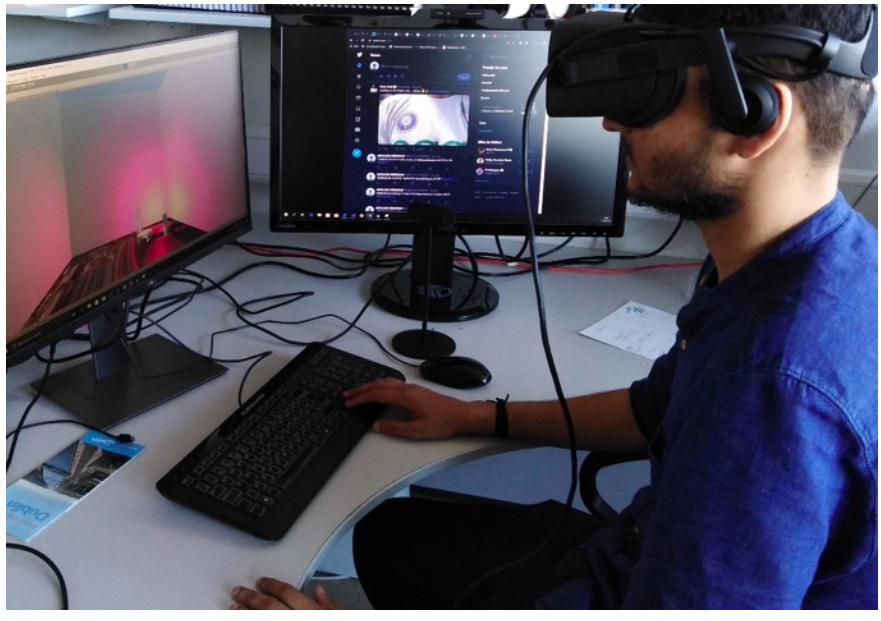

Fig. 7. Testbed testing

of $0.044 \mathrm{~s}$ (i.e. approximately $90 \%$ less delay than the Twitter API). MQTT usually performs better than REST due to the need of REST creating a new connection for every communication performed, while MQTT maintains connections alive.

\section{B. End-to-End Delay}

The end-to-end delay, which refers to the summed delays of posting and reading a tweet, was also measured ten times, and the minimum, average and maximum values were obtained. The plot presented in Fig. 9 illustrates these values.

This delay includes all combined times from a tweet being sent by the VR or Raspberry Pi to the action being executed on the Raspberry Pi or VR. The minimum, average and maximum delays observed were $0.6816 \mathrm{~s}, 0.8814 \mathrm{~s}$ and $1.4341 \mathrm{~s}$, respectively. 


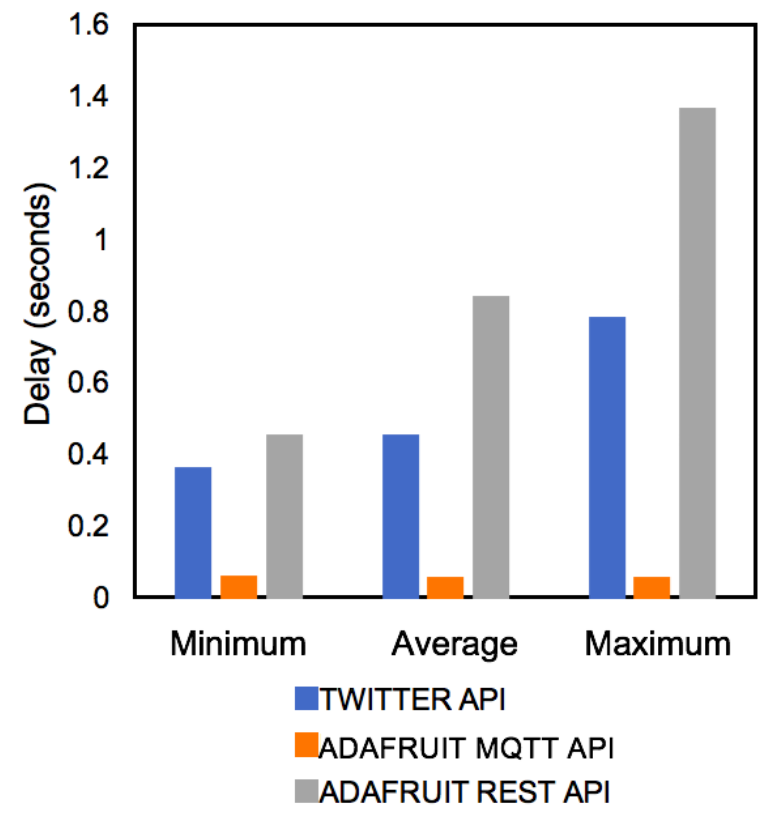

Fig. 8. Tweeting delay

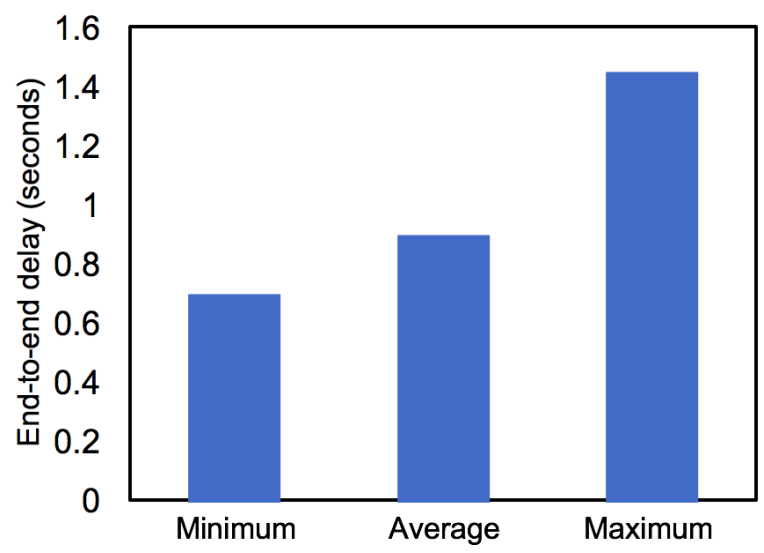

Fig. 9. End-to-end delay

\section{CONCLUSIONS AND Future WORK}

This paper presented the design, implementation and analysis of an integrated SIoT solution with VR and social media support. The application allows users to interact with IoT devices (represented by the Raspberry Pi) using VR headsets and Twitter.

The development of the applications for the Raspberry Pi and for the virtual environment was described, and these applications support two-way communications with Twitter, which is used as the cloud platform for users actions.

Tests indicated that the REST API provided by Twitter presents less communications delay than a baseline solution, however, MQTT communications which are not supported by the Twitter API could decrease the current delay.

The average end-to-end delay for actions in the platform is approximately $0.88 \mathrm{~s}$, which means that, for instance, an action performed in the VR environment will be tweeted and executed in the real device in less than one second. This demonstrates that Twitter is a feasible platform and interface for operating IoT devices.

Future work includes the addition of other IoT devices to the testbed, and tests with multiple simultaneous users using VR and Twitter. A Quality of Experience (QoE) study can also be conducted in order to assess the user perception of delays and latency. Support for additional IoT services can be added into the VR and SIoT platform as well, such as motion detection, control of appliances and a variety of alarms (e.g. gas, fire, water). The addition of other social network platforms, such as Facebook, is also considered for a thorough demonstration of the architecture, with a rich selection of integrated tools.

\section{ACKNOWLEDGEMENT}

This work was supported by the European Union's Horizon 2020 Research and Innovation programme under Grant Agreement no. 870610 for the TRACTION project. The support of the Science Foundation Ireland (SFI) Research Centres Programme Grant Numbers 12/RC/2289_P2 (Insight) and 16/SP/3804 (ENABLE) is gratefully acknowledged.

\section{REFERENCES}

[1] A. Al-Fuqaha, M. Guizani, M. Mohammadi, M. Aledhari, and M. Ayyash, "Internet of Things: A Survey on Enabling Technologies, Protocols and Applications," IEEE Communications Surveys \& Tutorials, vol. PP, no. 99, pp. 1-1, 2015. [Online]. Available: http://ieeexplore.ieee.org/lpdocs/epic03/wrapper.htm?arnumber=7123563

[2] C. Desogus, M. Anedda, M. Murroni, D. D. Giusto, and G. Muntean, "Remiot: Reputation-based network selection in multimedia iot," in 2019 IEEE Broadcast Symposium (BTS), 2019, pp. 1-6.

[3] B. Trinh, L. Murphy, and G. Muntean, "A reinforcement learningbased duty cycle adjustment technique in wireless multimedia sensor networks," IEEE Access, vol. 8, pp. 58774-58 787, 2020.

[4] A. Floris and L. Atzori, "Quality of Experience in the Multimedia Internet of Things: Definition and Practical Use-Cases," in Proc. of the IEEE International Conference on Communications Workshops (ICC Workshops), 2015, pp. 1747-1752.

[5] H. Xiao, C. Xu, T. Cao, S. Yang, L. Zhong, and G. Muntean, "Energy efficient for scalable video caching service over device-to-device communication," in 2019 15th International Wireless Communications Mobile Computing Conference (IWCMC), 2019, pp. 662-667.

[6] B. T. Nguyen, L. Murphy, and G. Muntean, "Enhanced scheme for adaptive multimedia delivery over wireless video sensor networks," in 2017 IEEE International Symposium on Broadband Multimedia Systems and Broadcasting (BMSB), 2017, pp. 1-7.

[7] A. A. Simiscuka and G.-M. Muntean, "Synchronisation between Real and Virtual-World Devices in a VR-IoT Environment," in Proc. of the IEEE International Symposium on Broadband Multimedia Systems, 2018, pp. 1-6.

[8] L. Atzori, A. Iera, G. Morabito, and M. Nitti, "The social internet of things (SIoT) - When social networks meet the internet of things: Concept, architecture and network characterization," Computer Networks, vol. 56, no. 16, pp. 3594-3608, 2012.

[9] D. Sheridan, A. Augusto Simiscuka, and G. Muntean, "Design, implementation and analysis of a twitter-based social iot network," in 2019 International Conference on Sensing and Instrumentation in IoT Era (ISSI), 2019, pp. 1-6.

[10] P. Cooper, "28 Twitter Statistics All Marketers Should Know in 2019," 2019. [Online]. Available: https://blog.hootsuite.com/twitter-statistics/ 
[11] M. A. Alharbe, "Awarenessability and influences on raising of traffic accidents through the content of social media in the internet of things: A practical empirical study by the internet of things and multimedia on university students in western Saudi Arabia," in International Conference on I-SMAC (IoT in Social, Mobile, Analytics and Cloud), I-SMAC 2018, 2018, pp. 48-51.

[12] F. Silva, D. Bogusevschi, and G. Muntean, "Innovative algorithms for prioritised ar/vr content delivery," in 2018 IEEE International Symposium on Broadband Multimedia Systems and Broadcasting (BMSB), 2018, pp. 1-5.

[13] Y. Han, Y. Ma, Y. Liao, and G. Muntean, "Qoe oriented adaptive streaming method for $360^{\circ}$ virtual reality videos," in 2019 IEEE SmartWorld, Ubiquitous Intelligence Computing, Advanced Trusted Computing, Scalable Computing Communications, Cloud Big Data Computing, Internet of People and Smart City Innovation (SmartWorld/SCALCOM/UIC/ATC/CBDCom/IOP/SCI), 2019, pp. 1655-1659.

[14] S. Abbas, A. A. Simiscuka, and G. M. Muntean, "A Platform Agnostic Solution for Inter-Communication between Virtual Reality Devices," in IEEE 5th World Forum on Internet of Things, WF-IoT 2019 - Conference Proceedings. Institute of Electrical and Electronics Engineers Inc., apr 2019, pp. 189-194.

[15] "Unity Real-Time Development Platform । 3D, 2D VR \& AR Visualizations." [Online]. Available: https://unity.com/

[16] "Oculus Rift: VR Headset for VR Ready PCs I Oculus." [Online]. Available: https://www.oculus.com/rift/\#oui-csl-rift-games=robo-recall

[17] "VIVE I Discover Virtual Reality Beyond Imagination." [Online]. Available: https://www.vive.com/eu/

[18] I. Toumpalidis, K. Cheliotis, F. Roumpani, and A. Hudson-Smith, "Vr binoculars: An immersive visualization framework for iot data streams," in Living in the Internet of Things: Cybersecurity of the IoT - 2018, March 2018, pp. 1-7.

[19] A. A. Simiscuka, T. M. Markande, and G.-M. Muntean, "Real-Virtual World Device Synchronisation in a Cloud-enabled Social Virtual Reality IoT Network," IEEE Access, vol. 7, pp. 106 588-106 599, 2019.

[20] A. Giordano, G. Spezzano, H. Sunarsa, and A. Vinci, "Twitter to integrate human and Smart Objects by a Web of Things architecture," in Proceedings of the 2015 IEEE 19th International Conference on Computer Supported Cooperative Work in Design, CSCWD 2015. Institute of Electrical and Electronics Engineers Inc., aug 2015, pp. 355-361.

[21] B. Jadhav and S. C. Patil, "Wireless Home monitoring using Social Internet of Things (SIoT)," in International Conference on Automatic Control and Dynamic Optimization Techniques, ICACDOT 2016, 2016, pp. 925-929.

[22] Waze, "Waze," 2020. [Online]. Available: https://www.waze.com/en$\mathrm{GB} /$

[23] "Twython - Twython 3.6.0 documentation," 2020. [Online]. Available: https://twython.readthedocs.io/en/latest/ 